OPEN ACCESS

Edited by: Inti Zlobec, University of Bern,

Switzerland

Reviewed by:

Parham Minoo,

University of Calgary,

Canada

Toru Furukawa,

Tokyo Women's Medical University,

Japan

*Correspondence:

Gisela Keller,

Institute of Pathology, Technische Universität München, Trogerstr. 18,

Munich D-81675, Germany

gisela.keller@/rz.tum.de

Specialty section:

This article was submitted to Gastrointestinal Cancers, a section of

the journal Frontiers in Oncology

Received: 23 February 2015

Accepted: 31 March 2015

Published: 22 April 2015

Citation:

Bauer L, Takacs A, Slotta-Huspenina

$J$, Langer $R$, Becker K, Novotny A, Ott K, Walch A, Hapfelmeier $A$ and Keller

$G$ (2015) Clinical significance of NOTCH1 and NOTCH2 expression in

gastric carcinomas: an immunohistochemical study.

Front. Oncol. 5:94.

doi: 10.3389/fonc.2015.00094

\section{Clinical significance of NOTCH1 and NOTCH2 expression in gastric carcinomas: an immunohistochemical study}

\begin{abstract}
Lukas Bauer ${ }^{1}$, Agnes Takacs ${ }^{1}$, Julia Slotta-Huspenina ${ }^{1}$, Rupert Langer ${ }^{2}$, Karen Becker ${ }^{1}$, Alexander Novotny ${ }^{3}$, Katja Ott ${ }^{4}$, Axel Walch ${ }^{5}$, Alexander Hapfelmeier ${ }^{6}$ and Gisela Keller ${ }^{\text {* }}$

${ }^{1}$ Institute of Pathology, Technische Universität München, Munich, Germany, ${ }^{2}$ Department of Pathology, University of Bern, Bern, Switzerland, ${ }^{3}$ Department of Surgery, Technische Universität München, Munich, Germany, ${ }^{4}$ Department of Surgery, Klinikum Rosenheim, Rosenheim, Germany, ${ }^{5}$ Institute of Pathology, Helmholtz-Zentrum München, Neuherberg, Germany, ${ }^{6}$ Institute of Medical Statistics and Epidemiology, Technische Universität München, Munich, Germany
\end{abstract}

Background: NOTCH signaling can exert oncogenic or tumor suppressive functions and can contribute to chemotherapy resistance in cancer. In this study, we aimed to clarify the clinicopathological significance and the prognostic and predictive value of $\mathrm{NOTCH} 1$ and $\mathrm{NOTCH} 2$ expression in gastric cancer (GC).

Methods: $\mathrm{NOTCH} 1$ and $\mathrm{NOTCH} 2$ expression was determined immunohistochemically in 142 primarily resected GCs using tissue microarrays and in 84 pretherapeutic biopsies from patients treated by neoadjuvant chemotherapy. The results were correlated with survival, response to therapy, and clinico-pathological features.

Results: Primarily resected patients with $\mathrm{NOTCH} 1$-negative tumors demonstrated worse survival. High NOTCH1 expression was associated with early-stage tumors and with significantly increased survival in this subgroup. Higher $\mathrm{NOTCH} 2$ expression was associated with early-stage and intestinal-type tumors and with better survival in the subgroup of intestinal-type tumors. In pretherapeutic biopsies, higher $\mathrm{NOTCH} 1$ and $\mathrm{NOTCH} 2$ expression was more frequent in non-responding patients, but these differences were statistically not significant.

Conclusion: Our findings suggested that, in particular, NOTCH1 expression indicated good prognosis in GC. The close relationship of high $\mathrm{NOTCH} 1$ and $\mathrm{NOTCH} 2$ expression with early tumor stages may indicate a tumor-suppressive role of $\mathrm{NOTCH}$ signaling in GC. The role of $\mathrm{NOTCH} 1$ and $\mathrm{NOTCH} 2$ in neoadjuvantly treated $\mathrm{GC}$ is limited.

Keywords: stomach neoplasms, receptor NOTCH1, receptor NOTCH2, prognosis, chemotherapy, immunohistochemistry

\section{Introduction}

The NOTCH signaling cascade is a highly conserved signaling pathway, with a crucial role in developmental processes and differentiation programs in various tissues. Aberrant NOTCH signaling has been implicated in carcinogenesis in various organs, and oncogenic, as well as tumor-suppressive functions, have been described $(1,2)$. In addition, there has been increasing 
evidence that NOTCH signaling contributes essentially to a drugresistant phenotype of tumor cells (3).

There are four NOTCH receptors (NOTCH1, 2, 3, 4), which can transmit activating signals from the cell membrane to the nucleus, leading then to the transcription of specific target genes $(1,2)$. Different and partly opposite roles in one tumor entity have been found for NOTCH1 and $\mathrm{NOTCH} 2$, indicating specific functions of the two receptors (4).

In gastric cancer (GC), NOTCH1 and NOTCH2 are believed to contribute to tumor progression (5-7). NOTCH1 has been reported to be a marker of poor prognosis, and increased expression of the NOTCH intracellular domain (NICD) was associated with the presence of lymph node metastasis and worse survival $(8,9)$.

In a recent study, we demonstrated the prognostic relevance of $\mathrm{NOTCH} 2$ gene expression in residual tumor cells after chemotherapy of neoadjuvantly treated GC patients. In addition, an increase in NOTCH2 expression in the residual tumor cells compared to the pretherapeutic biopsies was shown, indicating a prominent role of NOTCH2 in chemotherapy resistance in GC (10).

In this study, we first aimed to clarify the role of NOTCH1 and $\mathrm{NOTCH} 2$ protein expression in gastric carcinomas relative to prognosis and to the clinico-pathological characteristics of patients not treated by chemotherapy. Second, we aimed to determine the predictive and prognostic relevance of both proteins for neoadjuvant chemotherapy, and we included a group of pretherapeutic tumor biopsies of neoadjuvantly treated GC patients in the study.

\section{Materials and Methods}

\section{Patients and Specimens}

Gastric carcinomas from 226 patients operated on in the Department of Surgery at the Technische Universität München, Germany, between 1992 and 2010 were analyzed. The study population encompassed two subgroups and the patients' characteristics are shown in Table 1.

The first subgroup consisted of 142 resected specimens of patients who had been treated by surgical resection without neoadjuvant chemotherapy. Follow-up was calculated from the day of surgery until the last contact with the patient. Patients who died within 4 weeks after surgery were excluded from survival analysis. Overall survival (OS) was defined from the day of surgery to death from any cause. Survival data were available for 126 of the 142 patients. The median follow-up time for these patients was 40.1 months (range: $0.3-116.1$ months), and the median OS was 15.8 months (95\% CI 8.1-23.5 months).

The second subgroup encompassed 84 pretherapeutic biopsies of advanced gastric carcinoma patients (cT3 + cT4 only), who were treated with a platinum/5FU-based neoadjuvant chemotherapy, as reported previously (10).

Response to therapy was evaluated histopathologically, as described previously (11). In brief, all patients with $<10 \%$ residual tumor cells per tumor bed [tumor regression grade 1 (TRG1)] were classified as responders. All other patients with 10-50\% (TRG2) and with more than 50\% residual tumor cells per tumor bed (TRG3), as well as patients who demonstrated
TABLE 1 | Patient characteristics

\begin{tabular}{|c|c|c|c|}
\hline Variable & Category & $\begin{array}{c}\text { Primary resected } \\
\text { tumors } \\
n(\%)\end{array}$ & $\begin{array}{c}\text { Pretherapeutic } \\
\text { biopsies } \\
n(\%)\end{array}$ \\
\hline Patients & & $142(100)$ & $84(100)$ \\
\hline \multirow[t]{2}{*}{ Age (years) } & Median & 70.9 & 63.4 \\
\hline & Range & 39.5-99.9 & $39.2-77.6$ \\
\hline \multirow[t]{2}{*}{ Sex } & Female & 55 (39) & $23(27)$ \\
\hline & Male & $87(61)$ & $61(73)$ \\
\hline \multirow[t]{4}{*}{ Tumor localisation ${ }^{a}$} & Proximal & $33(23)$ & $59(70)$ \\
\hline & Medial & $39(27)$ & $14(17)$ \\
\hline & Distal & $54(38)$ & $10(12)$ \\
\hline & Total & $12(8)$ & $1(1)$ \\
\hline \multirow[t]{2}{*}{ Laurén classification } & Intestinal & $76(54)$ & $28(33)$ \\
\hline & Non-intestinal & $66(46)$ & $56(67)$ \\
\hline \multirow[t]{2}{*}{ Tumor grade } & $\mathrm{G} 1+2$ & $26(18)$ & $9(11)$ \\
\hline & $\mathrm{G} 3+4$ & $116(82)$ & 75 (89) \\
\hline \multirow[t]{2}{*}{ Resection category ${ }^{\mathrm{b}}$} & $\mathrm{RO}$ & $82(58)$ & $68(81)^{d}$ \\
\hline & $\mathrm{R} 1+2$ & $54(38)$ & $15(18)^{d}$ \\
\hline \multirow[t]{2}{*}{ pT category ${ }^{c}$} & $\mathrm{pT} 1+2$ & $64(45)$ & $63(75)^{\mathrm{d}, \mathrm{e}}$ \\
\hline & pT3 + 4 & $78(55)$ & $20(24)^{\mathrm{d}, \mathrm{e}}$ \\
\hline \multirow[t]{2}{*}{ pN category ${ }^{c}$} & pNO & $36(25)$ & $36(43)^{d, f}$ \\
\hline & $\mathrm{pN} 1+2+3$ & $106(75)$ & $47(56)^{d, f}$ \\
\hline
\end{tabular}

${ }^{a}$ For four patients, the tumor localization was unknown.

${ }^{b}$ For six patients, the resection category was unknown.

${ }^{\circ}$ TNM Classification of Malignant Tumors, 6th Edition, UICC (doi: 10.1371/journal.pone.0044566.t001).

${ }^{d}$ One patient with progressive disease was not submitted to surgery.

e Corresponds to ypT.

${ }^{f}$ Corresponds to ypN.

tumor progression during chemotherapy, were classified as nonresponders. Among the 84 patients, there were 22 (26\%) responding and 62 (74\%) non-responding patients.

Follow-up for these patients was calculated from the first day of chemotherapy until the last contact with the patient. OS was defined from the first day of chemotherapy until death from any cause. The median follow-up time for these patients was 40.6 months (range: 7.0-84.7 months), and the median OS was 50.6 months (mean OS 52.8 months 95\% CI: 50.0-60.7). The responders had significantly better OS than the non-responders ( $p_{\text {log-rank }}=0.041$; median OS not reached and 50.6 months, respectively; mean OS 63.9 months, $95 \% \mathrm{CI}$ : $50.0-77.7$ versus 44.3 months, 95\% CI 36.5-52.2, respectively).

The use of the tissue samples was approved by the local ethics committee of the Faculty of Medicine, Technische Universität München, Germany (Ref. 4071/11, 21/04/2011) and the informed consent of the patients was obtained according to the institutional regulations.

\section{Immunohistochemistry}

Immunohistochemistry was performed on formalin-fixed paraffin embedded (FFPE) tumor biopsies and on tissue microarrays (TMAs) of resected specimens. Tissue cores with a diameter of $1.0 \mathrm{~mm}$ from randomly selected tumor areas were used for the preparation of the TMAs. The monoclonal NOTCH1 
(bTAN20-s) and NOTCH2 antibodies (C651.6DbHN) were obtained from the Developmental Studies Hybridoma Bank (DSHB, the University of Iowa, Department of Biology, Iowa City, IA, USA) and were used undiluted for NOTCH1 and at a dilution of 1:30 for NOTCH2. Specificity of the antibodies was determined by western blotting, using the GC cell line MKN28 with short hairpin RNA-mediated knockdown of NOTCH1 or NOTCH2 expression, respectively (Figure S1 in Supplementary Material).

Expression was evaluated by two researchers (AT and RL for NOTCH2, LB and JSH for NOTCH1). Cytoplasmic and nuclear staining was determined separately. Negative, weak, medium, and strong staining intensities were scored as $0,1,2$, and 3 , respectively. The percentage of tumor cells with stained cytoplasm/nucleus was scored as 0 (negative), 1 ( $<10 \%), 2$ (10 to $<50 \%$ ), 3 (50 to $<80 \%$ ), or $4(\geq 80 \%)$. Multiplication of staining intensities and percentages resulted in a staining index (SI) ranging from 0 to 12, as described previously (12). We defined an SI of 0 as negative expression, $1-2$ as weak, 3-6 as moderate, and 8-12 as strong expression.

\section{Statistical Analysis}

Associations between the SIs and qualitative clinical and histopathological features were tested by the $\chi^{2}$ test or Fisher's exact test, depending on the cell counts of the corresponding contingency tables. Survival was estimated by Kaplan-Meier curves. The minimal $p$-value of log-rank tests was used to dichotomize the patients according to their various expression groups. Corresponding $p$-values served as effect measurements to determine optimality and should not interpreted in a probabilistic manner. For that purpose, appropriate $p$-values of maximally selected log-rank statistics were determined in a permutation test framework and are indicated as $p_{\max }$. In multivariate Cox regression analysis, stepwise forward variable selection was performed based on likelihood ratio tests. Because our study was an explorative study, there was no adjustment for multiple testing. All of the statistical testing was performed using a two-sided $5 \%$ significance level. SPSS software (IBM, Armonk, NY, USA), version 22.0, was used.

\section{Results}

\section{Frequency of NOTCH1 and NOTCH2 Expression in Primarily Resected Gastric Carcinomas}

NOTCH1 expression was evaluable in 133 of the 142 primarily resected tumor specimens. Strong cytoplasmic expression was found in $10(8 \%)$ of the 133 tumors, 38 (29\%) demonstrated moderate expression, 42 (32\%) demonstrated weak expression, and $43(32 \%)$ were negative.

NOTCH2 expression was evaluable in all of the 142 primarily resected tumors. Nine tumors $(6 \%)$ showed strong cytoplasmic expression, 61 (43\%) showed moderate cytoplasmic expression, $53(37 \%)$ showed weak cytoplasmic expression, and 19 (13\%) were negative.

None of the tumors showed nuclear NOTCH1 expression. Regarding nuclear NOTCH2 expression, 64 (45\%) of the 142 tumors were negative, 63 (44\%) demonstrated weak expression, and $15(11 \%)$ tumors showed moderate/strong expression.

The expression frequencies for primary resected tumors are shown in Figure 1A. Examples of staining patterns are shown in Figure 2.

\section{Expression of NOTCH1 and NOTCH2 in Primarily Resected Gastric Carcinomas and Correlation with Clinico-Pathological Characteristics}

Expression was analyzed for an association with the clinicopathological characteristics and with the survival of the patients in the subgroup of 142 primarily resected carcinomas. NOTCH1 was evaluable in 133 of the 142 cases and NOTCH2 in all 142 cases.

Strong NOTCH1 expression was associated with earlier tumor stages $\left(p=0.043 ; p_{\max }=0.061\right)$ (Figure 3A). No significant association of NOTCH1 expression with histopathological type according to Laurén, tumor differentiation, lymph node status, tumor location, or age was found.

Regarding NOTCH2, higher cytoplasmic expression was significantly associated with intestinal-type tumors $(p=0.004$; $\left.p_{\max }=0.011\right)($ Figure 3B), was more prevalent in lower tumor stages $(\mathrm{pT} 1+\mathrm{pT} 2)\left(p=0.030 ; p_{\max }=0.081\right)($ Figure $3 \mathrm{C})$ and was more frequently found in patients with negative lymph node

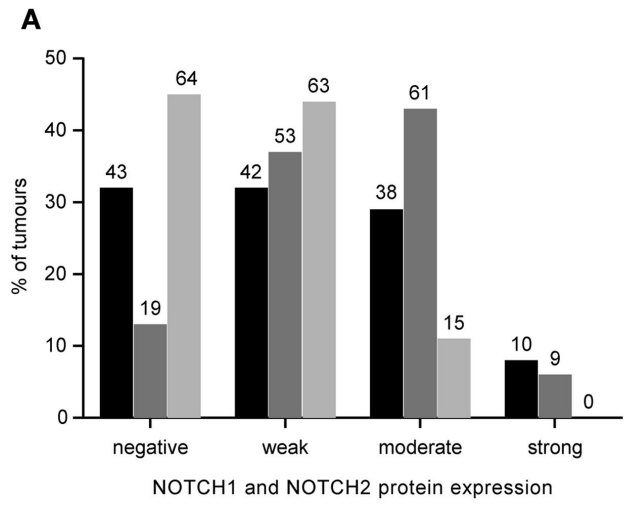

FIGURE 1 | Distribution of NOTCH1 and NOTCH2 protein expression in 142 primarily resected gastric carcinomas (A) and 84 pretherapeutic biopsies of neoadjuvantly treated patients (B). Staining indices are grouped

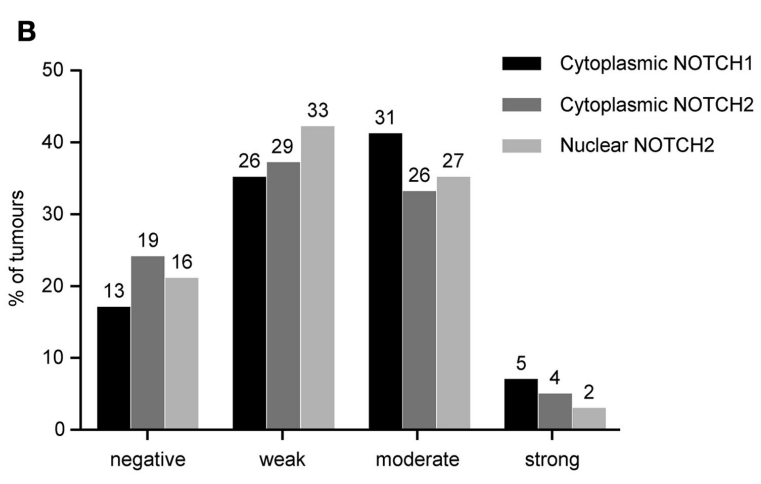

$\mathrm{NOTCH} 1$ and $\mathrm{NOTCH} 2$ protein expression

into negative, weak, moderate, and strong expression categories as described in the Section "Materials and Methods." The absolute number of cases is indicated above the bars. 


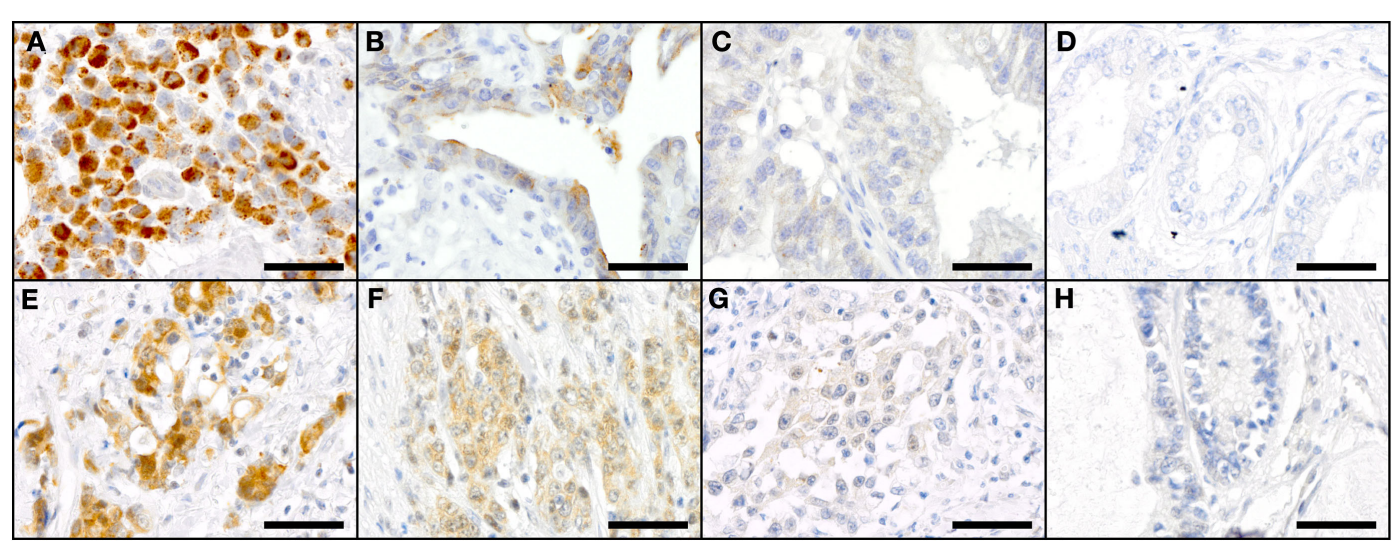

FIGURE 2 | Staining patterns for NOTCH1 (A-D) and NOTCH2

(E-H) in primary resected GC specimen. NOTCH1: (A) high-intensity cytoplasmic staining (SI 12), (B) medium-intensity cytoplasmic staining (SI 4), (C) low-intensity cytoplasmic staining (SI 2), and (D) negative cytoplasmic staining for $\mathrm{NOTCH} 1$ (SI 0); NOTCH2: (E) high-intensity cytoplasmic staining (SI 9) and low-intensity nuclear staining (SI 1), (F) medium-intensity cytoplasmic staining (SI 6) and low-intensity nuclear staining (SI 1); (G) low-intensity cytoplasmic staining (SI 3) and negative nuclear staining (SI 0); and $\mathbf{( H )}$ negative cytoplasmic and nuclear staining for $\mathrm{NOTCH} 2$. Scale bars: $50 \mu \mathrm{m}$.

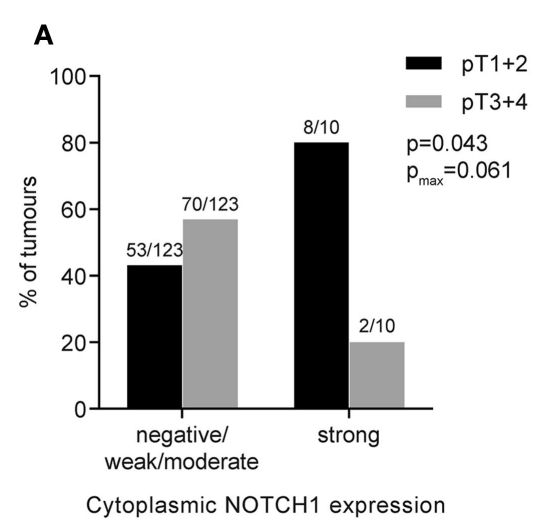

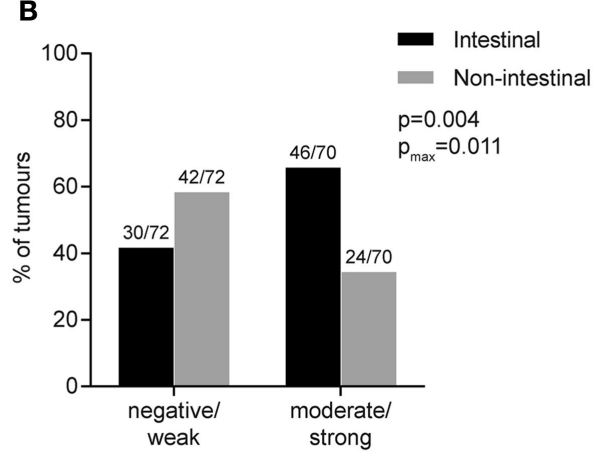

Cytoplasmic NOTCH2 expression

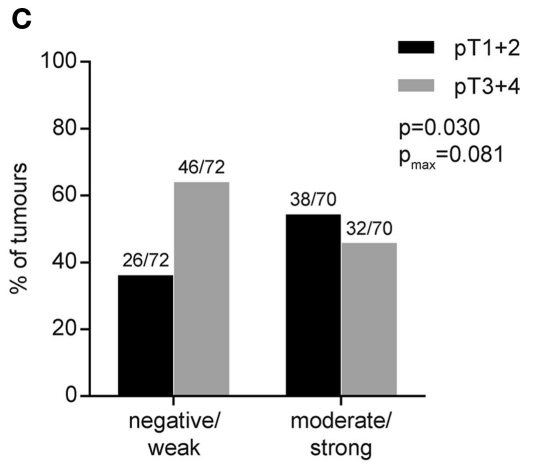

Cytoplasmic NOTCH2 expression

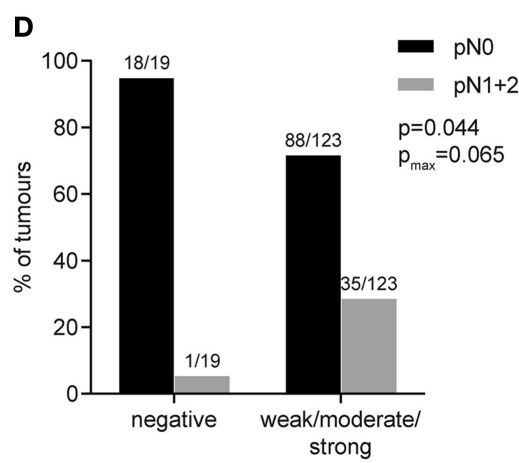

Cytoplasmic NOTCH2 expression

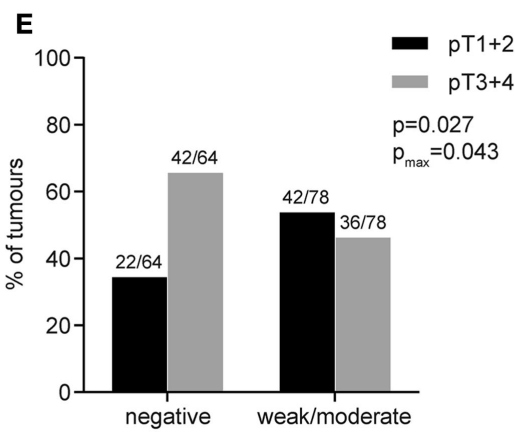

Nuclear NOTCH2 expression
FIGURE 3 | Association of NOTCH1 or NOTCH2 protein expression with clinico-pathological features of primary resected gastric carcinomas. Numbers above the bars indicate the absolute number of cases. $p$-Values were determined by the $\chi^{2}$-test or Fisher's exact test; $p_{\max }$ corresponds to $p$-values for maximally selected statistics. (A) Cytoplasmic NOTCH1 expression and association with tumor stage; (B) cytoplasmic NOTCH2 expression and association with histopathological tumor type; (C) cytoplasmic NOTCH2 expression and association with tumor stage; (D) cytoplasmic NOTCH2 expression and association with lymph node status; (E) nuclear NOTCH2 expression and association with tumor stage. status $\left(p=0.044 ; p_{\max }=0.065\right)$ (Figure 3D). Regarding nuclear NOTCH2 expression, positive expression (weak/moderate/ strong) was associated with lower tumor stages $(\mathrm{pT} 1+\mathrm{pT} 2)$, compared to advanced tumor stages (pT3 + pT4) $(p=0.027$; $\left.p_{\max }=0.043\right)$ (Figure 3E). No other significant differences were found. 


\section{Expression of NOTCH1 and NOTCH2 in Primarily Resected Gastric Carcinomas and Prognosis}

Patients who did not show any NOTCH1 expression in their tumors demonstrated significantly worse survival, compared to all other patients having weak, moderate, or strong NOTCH1 expression $\left(p_{\text {log-rank }}=0.035 ; p_{\max }=0.117\right)($ Figure 4A).

Analyzing the patients with NOTCH1-negative tumors for an association with survival in the subgroups of (a) early $(\mathrm{pT} 1+\mathrm{pT} 2)$ and advanced (pT3+pT4) tumor stages separately, (b) in intestinal and non-intestinal-type tumors, and (c) in completely resected (R0) tumors only, revealed significant associations with NOTCH1-negative tumors and worse survival in patients with early tumor stages but not in patients with advanced tumors ( $p_{\text {log-rank }}=0.007$ and $p_{\text {log-rank }}=0.999$, respectively) (Figures 4B,C). A trend toward an association of these patients with worse survival was observed in nonintestinal tumors but not in intestinal tumors $\left(p_{\text {log-rank }}=0.055\right.$ and $p_{\text {log-rank }}=0.449$, respectively) (Figures $4 \mathrm{D}, \mathrm{E}$ ). In addition, patients with NOTCH1-negative tumors showed a significant decrease in survival in the subgroup of patients with completely resected tumors $\left(p_{\text {log-rank }}=0.048\right)$ (Figure $\left.4 F\right)$.

Multivariate Cox regression analysis, including NOTCH1 expression and the standard prognostic factors, namely $\mathrm{pT}, \mathrm{pN}$, and R-status, revealed no prognostic relevance for NOTCH1 expression neither in the whole study group nor in the subgroup of completely resected patients. In the subgroup of patients with early tumor stages $(n=64)$, NOTCH1 expression was the second most important factor (HR: 0.82, 95\% CI: 0.67-0.99, $p=0.038$ ) after lymph node metastasis (HR: 3.35, 95\% CI 1.30-8.68, $p=0.013)$.

Considering NOTCH2, cytoplasmic or nuclear expression did not demonstrate an association with survival. Subgroup analyses showed an association of strong cytoplasmic NOTCH2 expression with increased survival in patients with intestinaltype tumors $\left(p_{\text {log-rank }}=0.043 ; p_{\max }=0.060\right.$ ) (Figure 5A). In addition, moderate/high nuclear NOTCH2 expression was also significantly associated with better survival in this subgroup $\left(p_{\text {log-rang }}=0.039 ; p_{\max }=0.020\right)$ (Figure 5B). Multivariate analysis in intestinal tumors indicated R-status (HR: 2.78, 95\% CI: $1.39-5.55, p=0.004$ ) and tumor stage (HR: $2.61,95 \% \mathrm{CI}$ : $1.28-5.30, p=0.008)$ as independent prognostic factor but not NOTCH2 expression.

\section{Frequency of NOTCH1 and NOTCH2 Expression in Pretherapeutic Biopsies of Neoadjuvantly Treated Patients}

Expression of NOTCH1 was evaluable in 75 of 84 pretherapeutic biopsies of patients treated with a neoadjuvant chemotherapy.

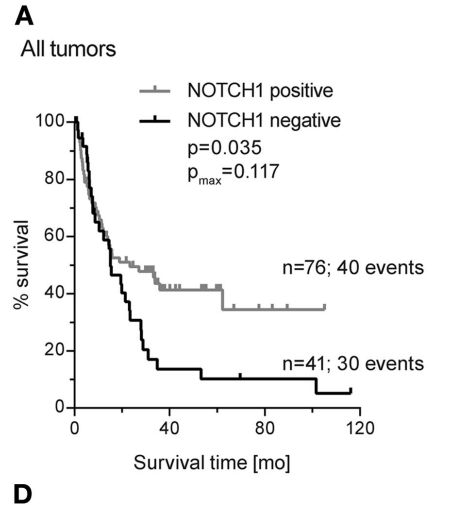

Non-intestinal type tumors

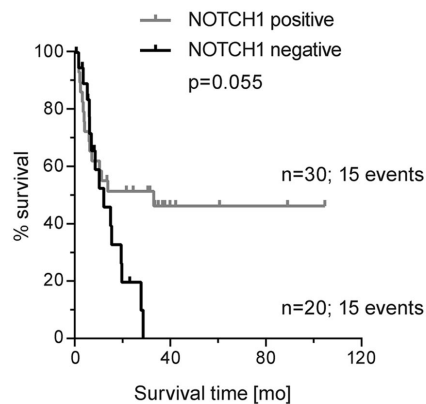

B

pT1+2

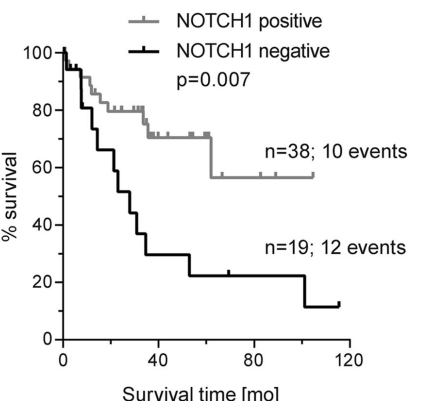

E

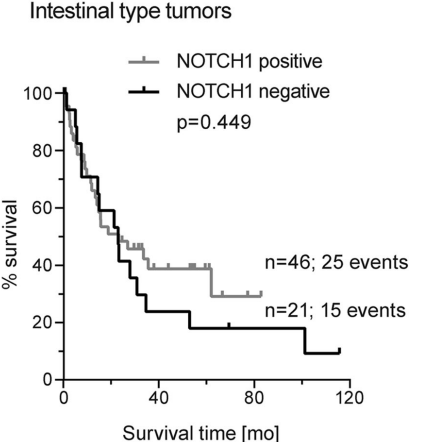

pT3+4

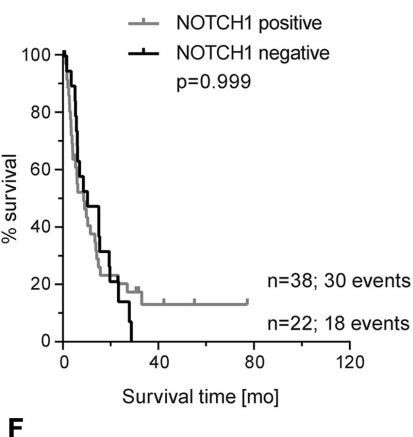

R0-resected tumors

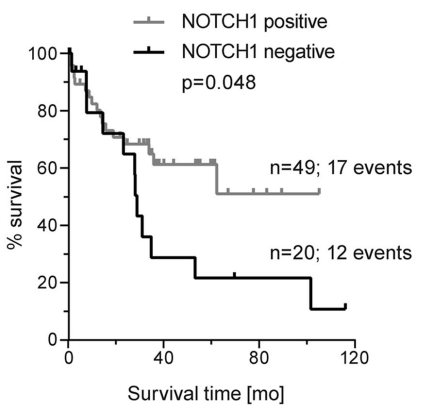

FIGURE 4 | Kaplan-Meier survival curves for NOTCH1 protein expression in primary resected gastric carcinomas. (A) All tumors (median OS: 15.1 months; $95 \% \mathrm{Cl} 8.3-22.0$ versus 23.3 months; $95 \% \mathrm{Cl}$ 4.6-26.1); (B) subgroup of early-stage tumors (pT1 + 2) (median OS: 28.0 months; $95 \%$ Cl 16.2-40.0 versus median OS not reached); (C) subgroup of advanced-stage tumors (pT3 + 4) (median OS: 10.3 months; 95\% Cl 0-21.8 versus 8.7 months; $95 \%$ Cl 3.1-14.3); (D) subgroup of non-intestinal-type tumors (median OS: 12.2 months; 95\% Cl 4.1-20.2 versus 33.2 months; 95\% Cl not applicable); (E) subgroup of intestinal-type tumors (median OS: 23.1 months; 95\% Cl 12.0-34.2 versus 23.3 months; 95\% Cl 3.0-43.6); (F) subgroup of patients with completely resected tumors (R0) (median OS: 28.8 months; 95\% Cl 27.1-30.5 versus median OS not reached); $p$-values were determined by log-rank statistics; $p_{\max }$. corresponds to $p$-values for maximally selected statistics. 


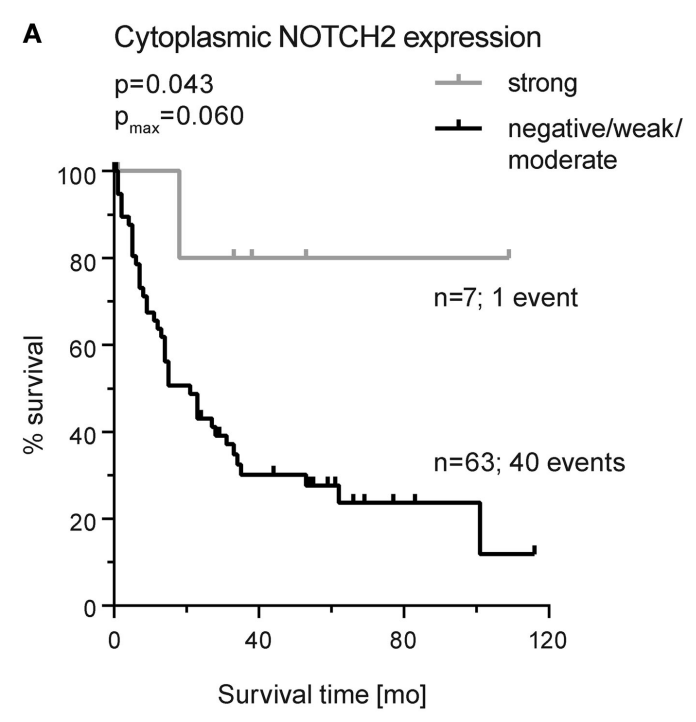

B Nuclear $\mathrm{NOTCH} 2$ expression

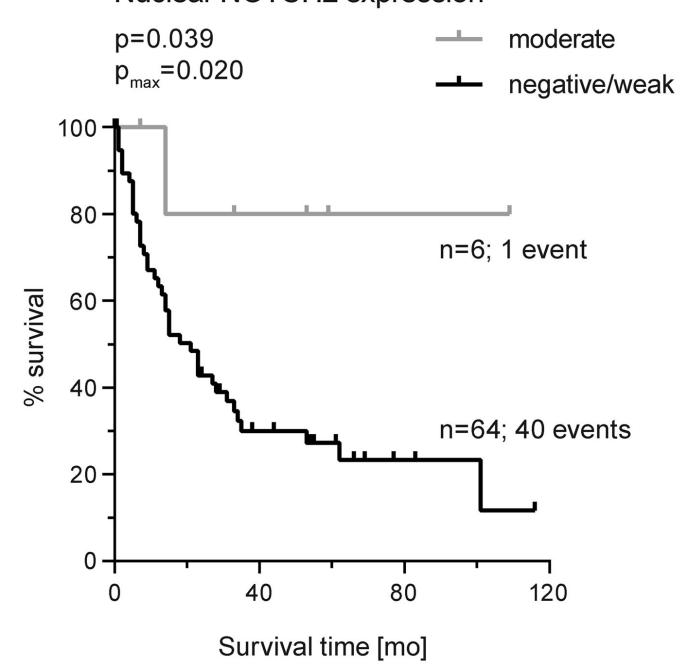

FIGURE 5 | Kaplan-Meier survival curves for NOTCH2 protein expression in primary resected gastric carcinomas of the intestinal type. (A) Cytoplasmic expression (median OS: not reached versus 21.2 months; $95 \% \mathrm{Cl} 11.4-31.3$ ) and (B) nuclear expression (median OS: not reached versus 21.4 months; $95 \% \mathrm{Cl} 11.6-31.1)$. $p$-Values were determined by log-rank statistics; $p_{\max }$ corresponds to $p$-value for maximally selected statistics.

Thirteen (17\%) of the 75 samples were negative for NOTCH1 protein expression, 26 (35\%) showed a weak cytoplasmic expression, 31 (41\%) showed a moderate expression, and 5 (7\%) demonstrated a strong cytoplasmic NOTCH1 expression. None of the samples showed nuclear NOTCH1 expression.

NOTCH2 was evaluable in 78 of the 84 samples. Cytoplamic NOTCH2 expression was strong in $4(5 \%)$, moderate in $26(33 \%)$, weak in 29 (37\%), and negative in $19(24 \%)$ of these samples. Nuclear NOTCH2 expression was observed to be strong in $2(3 \%)$, moderate in 27 (35\%), weak in 33 (42\%), and negative in 16 (21\%) of pretherapeutic biopsies. The expression frequencies for pretherapeutic biopsies are shown in Figure 1B.
TABLE 2|NOTCH1 and NOTCH2 protein expression in pretherapeutic tumor biopsies and response to neoadjuvant chemotherapy.

\begin{tabular}{|c|c|c|c|c|c|c|}
\hline & \multicolumn{3}{|c|}{ NOTCH1 } & \multicolumn{3}{|c|}{ NOTCH2 } \\
\hline & $S \begin{array}{c}n \\
\left.S\right|^{a}<4\end{array}$ & $\begin{array}{c}n \\
S I \geq 4\end{array}$ & $p$-value ${ }^{b}$ & $\begin{array}{c}n \\
\mathrm{SI}<3\end{array}$ & $\begin{array}{c}n \\
\mathrm{sI} \geq 3\end{array}$ & $p$-value ${ }^{b}$ \\
\hline Responders & 18 & 1 & 0.056 & 19 & 2 & 0.079 \\
\hline Non-responders & 41 & 15 & & 40 & 17 & \\
\hline Total & 59 & 16 & & 59 & 19 & \\
\hline
\end{tabular}

${ }^{a}$ Staining index.

${ }^{b}$ Fisher's exact test.

\section{Expression of NOTCH1 and NOTCH2 in Pretherapeutic Biopsies and Response to Therapy}

No association of NOTCH1 or NOTCH2 expression with response was found using the classification system of negative (SI 0), weak (SI 1-2), moderate (SI 3-6), and strong (SI 8-12) expression. Searching for an optimal cut-off value to differentiate responding and non-responding patients revealed a trend toward an association of tumors with higher cytoplasmic NOTCH1 $(\mathrm{SI} \geq 6)$ or $\mathrm{NOTCH} 2(\mathrm{SI} \geq 4)$ expression with worse response ( $p=0.056$ and $p=0.079$, respectively) (Table 2 ). No significant associations with survival were found.

\section{Discussion}

In this study, we addressed the potential clinical impact of NOTCH1 and NOTCH2 expression in GC by analyzing both proteins in a group of patients treated directly by primary resection of their tumors and in another group treated with platinum/5FUbased neoadjuvant chemotherapy.

One of the most interesting results of our study was the finding of higher expression of cytoplasmic NOTCH1 and of cytoplasmic and nuclear NOTCH2 in early tumor stages (pT1 + pT2), compared to advanced stages (pT3 + pT4). This finding indicated that a decrease in expression of both proteins occurred during tumor progression, suggesting a tumor-suppressive function of NOTCH signaling in gastric carcinogenesis. Furthermore, our study showed the prognostic relevance of NOTCH1, with higher expression being associated with increased survival and no lymph node metastasis. Beyond this, an impressive prognostic role for NOTCH1 was found for patients with T1/T2 tumors but not for patients with advanced pT3/pT4 tumors. Patients with higher NOTCH1-expressing tumors in the subgroup with early pathological tumor stages had significantly better survival than those with a low expression, and multivariate analysis indicated that NOTCH1 expression was an independent prognostic factor in this subgroup. This finding characterized NOTCH1 expression as a prognostic factor, particularly during early tumor development, and it additionally supported a possible tumor-suppressive role for NOTCH signaling.

It is well known that NOTCH signaling can exert oncogenic or tumor-suppressive functions and that individual NOTCH receptors can exert opposite functions in context- and time-dependent manners, even in the same tumor type (2). For example, a tumor-suppressive function has been reported for skin and 
liver cancers, an oncogenic role for $\operatorname{NSCLC}(2,13)$ and a dual role in the same tumor type for colorectal and pancreatic cancers $(4,14)$. The findings of our study ascribed a tumor-suppressive role to NOTCH signaling in GC. This result was essentially in agreement with a functional analysis of $\mathrm{NOTCH} 2$ in a GC cell line, which demonstrated that downregulation of NOTCH2 by siRNA enhanced tumor cell invasion (15). However, our results were in contrast to findings from other studies mainly performed in Asian countries, which have reported a contribution of higher expression of NOTCH1 or NOTCH2 to tumor progression and, in particular, an association of higher expression of NOTCH1 with worse prognosis $(5,7-9,16)$. Furthermore, higher NOTCH2 expression was more frequently found in intestinal-type tumors in our study, again inconsistently with an analysis of Asian patients reporting no associations of NOTCH2 expression with histopathological type (6). The reasons for these discrepancies are not clear, but they could be related to differences in the study populations, in the analytical technologies or in the applied scoring methods. In addition, differences related to the specificity of the antibodies might exist. In this context, we would like to emphasize that we demonstrated the specificity of the antibodies that we used in our study by western blotting. In addition, based on the results in the respective NOTCH1- and NOTCH2knockdown cells, we showed that cross-reaction of both antibodies was very unlikely. Interestingly, we observed positive nuclear staining only for NOTCH2 and not for NOTCH1. This finding might reflect varying degradation rates of nuclear NOTCH1 and $\mathrm{NOTCH} 2$ proteins or variations in the sensitivities of the specific antibodies.

In the subgroup of neoadjuvantly treated GC patients, we identified only a trend toward an association of a higher NOTCH1 and $\mathrm{NOTCH} 2$ expression in the pretherapeutic biopsies and worse tumor regression after chemotherapy. Recent studies have indicated an association between NOTCH1 or NOTCH3 and cisplatin resistance in squamous head and neck and ovarian carcinomas and in EBV-associated naso-pharynx carcinomas, respectively (17-19).

\section{References}

1. Andersson ER, Sandberg R, Lendahl U. NOTCH signaling: simplicity in design, versatility in function. Development (2011) 138:3593-612. doi:10.1242/dev. 063610

2. Ranganathan P, Weaver KL, Capobianco AJ. NOTCH signaling in solid tumors: a little bit of everything but not all the time. Nat Rev Cancer (2011) 11:338-51. doi:10.1038/nrc3035

3. Wang Z, Li Y, Ahmad A, Azmi AS, Banerjee S, Kong D, et al. Targeting NOTCH signaling pathway to overcome drug resistance for cancer therapy. Biochim Biophys Acta (2010) 1806:258-67. doi:10.1016/j.bbcan.2010.06.001

4. Mazur PK, Einwächter H, Lee M, Sipos B, Nakhai H, Rad R, et al. NOTCH2 is required for progression of pancreatic intraepithelial neoplasia and development of pancreatic ductal adenocarcinoma. Proc Natl Acad Sci U S A (2010) 107:13438-43. doi:10.1073/pnas.1002423107

5. Yeh TS, Wu CW, Hsu KW, Liao WJ, Yang MC, Li AF, et al. The activated NOTCH1 signal pathway is associated with gastric cancer progression through cyclooxygenase-2. Cancer Res (2009) 69:5039-48. doi:10.1158/ 0008-5472.CAN-08-4021

6. Sun Y, Gao X, Liu J, Kong QY, Wang XW, Chen XY, et al. Differential NOTCH1 and NOTCH2 expression and frequent activation of NOTCH
We are aware that our study had limitations, mainly related to its retrospective nature and to the limited number of tumors we analyzed. Thus, further studies are needed to delineate in greater detail the role of NOTCH1 and NOTCH2 expression in gastric carcinogenesis and the impact of these molecules in the prediction of response to neoadjuvant chemotherapy in GC. Furthermore, functional analyses are needed to clarify a possible tumor-suppressive role of NOTCH signaling in GC.

Nevertheless, in conclusion and to the best of our knowledge, our study showed for the first time a close relationship of a decrease of NOTCH1 and NOTCH2 expression with tumor progression in GC, and our findings suggested that, in particular, NOTCH1 expression was a marker for good prognosis. These findings taken together may suggest a tumor-suppressive role of the NOTCH signaling pathway in GC.

\section{Author Contributions}

Study design and writing of the manuscript (LB and GK); immunostaining (LB and AT), data analysis and data interpretation (LB, AT, JS-H, RL, AH, and GK); contribution of clinical data and material (KB, $\mathrm{KO}, \mathrm{AN}$, and $\mathrm{AW})$; critical revising and final approvement of the manuscript (all authors); agreement to be accountable for all aspects of the work in ensuring that questions related to the accuracy or integrity of any part of the work are appropriately investigated and resolved (all authors).

\section{Acknowledgments}

This study was supported by the Wilhelm-Sander Stiftung under grant number 2011.017.1 to GK and RL.

\section{Supplementary Material}

The Supplementary Material for this article can be found online at http://journal.frontiersin.org/article/10.3389/fonc.2015.00094/ abstract

signaling in gastric cancers. Arch Pathol Lab Med (2011) 135:451-8. doi:10. 1043/2009-0665-OA.1

7. Tseng YC, Tsai YH, Tseng MJ, Hsu KW, Yang MC, Huang KH, et al. NOTCH2induced COX-2 expression enhancing gastric cancer progression. Mol Carcinog (2012) 51:939-51. doi:10.1002/mc.20865

8. Zhang $\mathrm{H}$, Wang $\mathrm{X}, \mathrm{Xu}$ J, Sun Y. NOTCH1 activation is a poor prognostic factor in patients with gastric cancer. Br J Cancer (2014) 110:2283-90. doi:10.1038/ bjc. 2014.135

9. Luo DH, Zhou Q, Hu SK, Xia YQ, Xu CC, Lin TS, et al. Differential expression of NOTCH1 intracellular domain and $\mathrm{p} 21$ proteins, and their clinical significance in gastric cancer. Oncol Lett (2014) 7:471-8. doi:10.3892/ol.2013.1751

10. Bauer L, Langer R, Becker K, Hapfelmeier A, Ott K, Novotny A, et al. Expression profiling of stem cell-related genes in neoadjuvant-treated gastric cancer: a NOTCH2, GSK3B and beta-catenin gene signature predicts survival. PLoS One (2012) 7:e44566. doi:10.1371/journal.pone.0044566

11. Becker K, Langer R, Reim D, Novotny A, Meyer zum Buschenfelde C, Engel $\mathrm{J}$, et al. Significance of histopathological tumor regression after neoadjuvant chemotherapy in gastric adenocarcinomas: a summary of 480 cases. Ann Surg (2011) 253:934-9. doi:10.1097/SLA.0b013e318216f449

12. Langer R, Mutze K, Becker K, Feith M, Ott K, Höfler H, et al. Expression of class I histone deacetylases (HDAC1 and HDAC2) in oesophageal adenocarcinomas: 
an immunohistochemical study. J Clin Pathol (2010) 63:994-8. doi:10.1136/jcp. 2010.080952

13. Lobry C, Oh P, Aifantis I. Oncogenic and tumor suppressor functions of NOTCH in cancer: it's NOTCH what you think. J Exp Med (2011) 208:1931-5. doi:10.1084/jem.20111855

14. Chu D, Zheng J, Wang W, Zhao Q, Li Y, Li J, et al. NOTCH2 expression is decreased in colorectal cancer and related to tumor differentiation status. Ann Surg Oncol (2009) 16:3259-66. doi:10.1245/s10434-009-0655-6

15. Guo LY, Li YM, Qiao L, Liu T, Du YY, Zhang JQ, et al. NOTCH2 regulates matrix metallopeptidase 9 via PI3K/AKT signaling in human gastric carcinoma cell MKN-45. World J Gastroenterol (2012) 18:7262-70. doi:10.3748/wjg.v18. i 48.7262

16. Li DW, Wu Q, Peng ZH, Yang ZR, Wang Y. (Expression and significance of NOTCH1 and PTEN in gastric cancer). Ai Zheng (2007) 26:1183-7.

17. Gu F, Ma Y, Zhang Z, Zhao J, Kobayashi H, Zhang L, et al. Expression of Stat 3 and NOTCH1 is associated with cisplatin resistance in head and neck squamous cell carcinoma. Oncol Rep (2010) 23:671-6.

18. Park JT, Chen X, Trope CG, Davidson B, Shih IeM, Wang TL. NOTCH3 overexpression is related to the recurrence of ovarian cancer and confers resistance to carboplatin. Am J Pathol (2010) 177:1087-94. doi:10.2353/ajpath. 2010.100316

19. Man CH, Wei-Man Lun S, Wai-Ying Hui J, To KF, Choy KW, Wing-Hung Chan A, et al. Inhibition of NOTCH3 signaling significantly enhances sensitivity to cisplatin in EBV-associated nasopharyngeal carcinoma. J Pathol (2012) 226:471-81. doi:10.1002/path.2997

Conflict of Interest Statement: The authors declare that the research was conducted in the absence of any commercial or financial relationships that could be construed as a potential conflict of interest. The Associate Editor Inti Zlobec declares that, despite having collaborated with author Rupert Langer, the review process was handled objectively and no conflict of interest exists.

Copyright (C) 2015 Bauer, Takacs, Slotta-Huspenina, Langer, Becker, Novotny, Ott, Walch, Hapfelmeier and Keller. This is an open-access article distributed under the terms of the Creative Commons Attribution License (CC BY). The use, distribution or reproduction in other forums is permitted, provided the original author(s) or licensor are credited and that the original publication in this journal is cited, in accordance with accepted academic practice. No use, distribution or reproduction is permitted which does not comply with these terms. 